\title{
THE RESISTANCE TO FIBRINOLYTIC ACTIVITY OF THE HEMO- LYTIC STREPTOCOCCUS WITH SPECIAL REFERENCE TO PATIENTS WITH RHEUMATIC FEVER AND RHEUMATOID (ATROPHIC) ARTHRITIS ${ }^{1}$
}

\author{
By WALTER K. MYERS, CHESTER S. KEEFER AND WILLIAM F. HOLMES, JR. \\ (From the Thorndike Memorial Laboratory, Second and Fourth Medical Services (Harvard), \\ Boston City Hospital and the Department of Medicine, Harvard Medical School, Boston)
}

(Received for publication September 11, 1934)

Recently Tillett and Garner (1) have demonstrated that broth cultures of hemolytic streptococci of human origin rapidly liquefy the fibrinclot of human plasma. Cultures of other species of bacteria obtained from human beings fail to exhibit this property. Tillett, Edwards and Garner (2) further found that the plasma clot from patients convalescent from acute hemolytic streptococcal infections was highly resistant to the action of broth cultures of a human hemolytic streptococcus. The observations of Tillett and his coworkers were confirmed by Hadfield, Magee and Perry (3).

Previously, we $(4,5,6)$ have studied groups of patients with rheumatic fever and rheumatoid arthritis to determine what, if any, relationship exists between streptococcal infection and these diseases. In continuing these studies, we have determined the resistance to fibrinolysis of the blood plasma of patients with rheumatic fever and rheumatoid arthritis and of control groups of patients.

\section{METHODS OF STUDY}

All patients were studied while in the hospital. The methods employed in the determination of the resistance to fibrinolysis of blood plasma followed closely the method described by Tillett and his coworkers $(1,2)$.

At weekly intervals 5 cc. of blood were collected. Potassium oxalate, 0.01 gram per $5 \mathrm{cc}$. of blood, was employed as an anticoagulant. The plasma was separated by centrifugalization and was used within two hours after its withdrawal.

A strain of the hemolytic streptococcus supplied by Dr. Tillett and designated as $\mathrm{CO}$ was employed in all the tests. Freshly grown 18 to 24-

1 This investigation was aided in part by a grant from the Proctor Fund of the Harvard Medical School for the Study of Chronic Diseases. hour cultures of this organism were grown in veal muscle infusion broth, adjusted to a $\mathrm{pH}$ of 7.2 and containing one per cent of peptone and 0.2 per cent of $\mathrm{Na}_{2} \mathrm{HPO}_{4}$.

To 0.2 cc. of fresh oxalated plasma was added $0.8 \mathrm{cc}$. of sterile physiological salt solution. To this $0.5 \mathrm{cc}$. of a fresh broth culture of the $\mathrm{CO}$ strain of the hemolytic streptococcus was added and well mixed. Then $0.25 \mathrm{cc}$. of a 0.25 per cent sterile solution of $\mathrm{CaCl}_{2}$ was added and well mixed. The tubes were placed immediately in a water bath at $37.5^{\circ} \mathrm{C}$. With repeated observations the times of solid coagulation and, finally, complete dissolution of the clot were recorded. All tests in which the plasma clot was resistant to dissolution after 24 hours' incubation were arbitrarily terminated.

The classification of the degree of resistance of the plasma clot as suggested by Tillett, Edwards and Garner (2) was followed. The highest degree of resistance shown by a patient's plasma during the period of observation was utilized in the classification shown in Table I.

\section{RESULTS}

Determinations of the resistance to fibrinolysis of 520 samples of plasma from 135 individuals were made. Their ages varied between thirteen and seventy years. No correlation could be established between the age of the individual and the ability to develop resistance to fibrinolysis.

The plasmas from groups of individuals without evidence of hemolytic streptococcal infection were studied. Samples of plasma from fourteen apparently normal laboratory workers were followed at frequent intervals for several months. A group of twenty-two patients with pulmonary tuberculosis, lobar pneumonia, herpes zoster and lung abscess were studied on fifty-one occasions. 
TABLE I

Results of fibrinolytic tests with the plasma clot

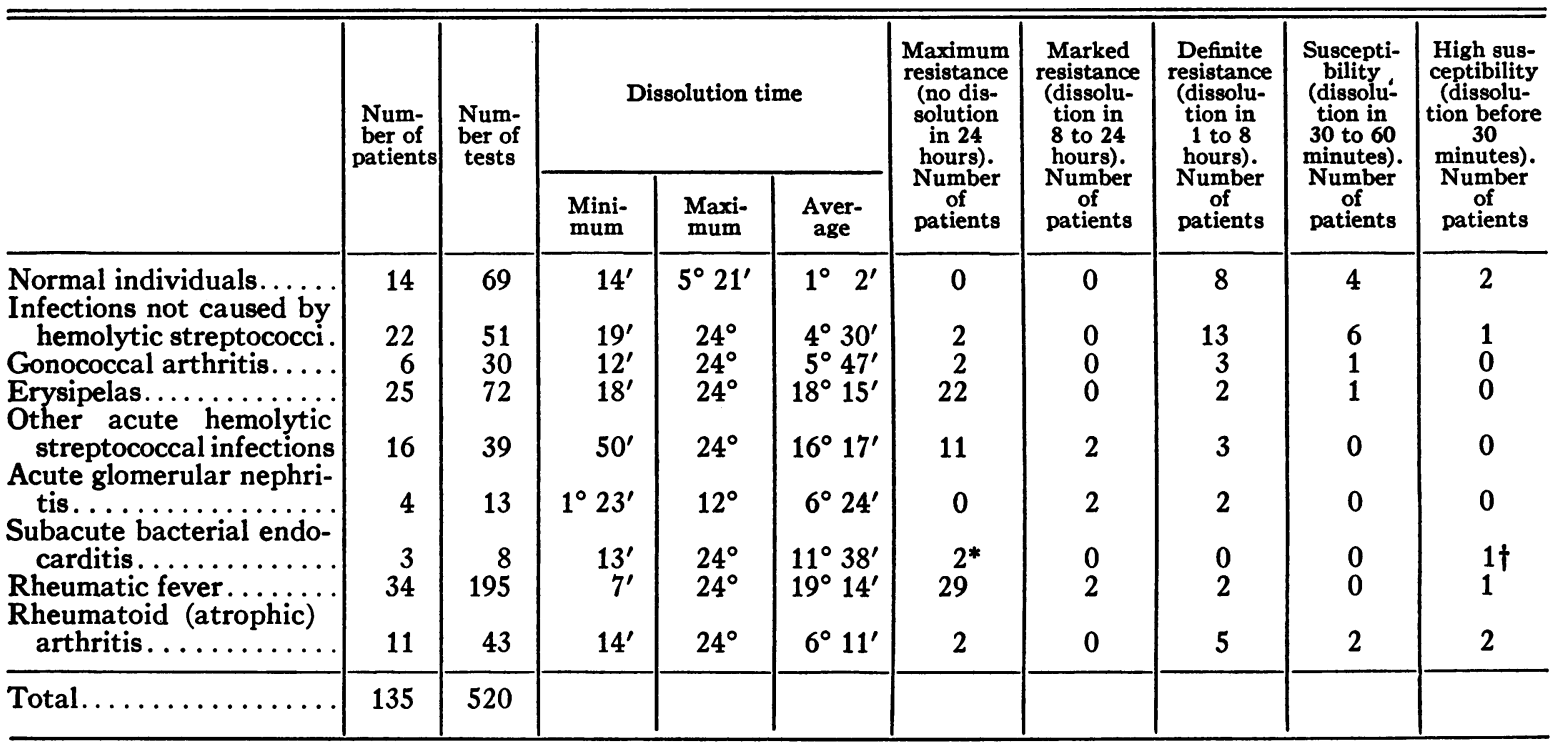

* Associated with Streptococcus viridans.

$\dagger$ Associated with an indifferent streptococcus.

In addition, thirty samples of plasma from six patients with gonococcal arthritis were tested. The results of the tests of the plasma of these forty-two individuals are summarized in Table I.

From time to time relatively small variations were noted in the time of dissolution of the plasma clot of normal individuals. Such fluctuations were probably due to variations in the concentration of the fibrinolysin in the cultures. The employment of samples of plasma from the same individual repeatedly in each set of tests served as a satisfactory control. It was unusual to obtain a result which showed more than "definite resistance" to fibrinolysis in the control group. In but four, or 9.5 per cent, of the whole group of individuals without evidence of infection with the hemolytic streptococcus did any samples of plasma show great resistance to fibrinolysis.

For comparison with normal individuals and those with rheumatic fever and rheumatoid arthritis, the effect of acute infection with the hemolytic streptococcus on the resistance to fibrinolysis was studied. In Table $I$ is a summary of the results of seventy-two tests done on the plasma from sixteen patients with other acute hemolytic streptococcal infections such as acute tonsillitis and acute otitis media.

"Maximum resistance" and "marked resist-

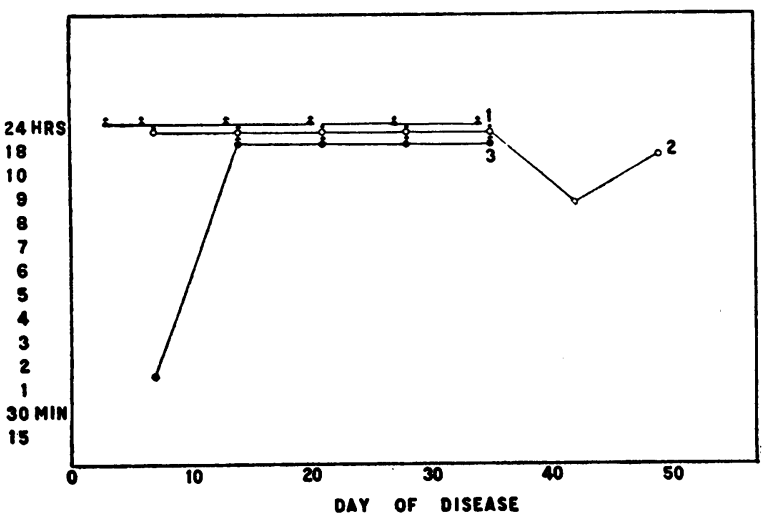

Fig. 1. The Changes in the Dissolution Time of the Plasma Clot of Three Patients with ErySIPELAS.

ance" was encountered in thirty-three, or 80.5 per cent, of these forty-one patients. Changes in the resistance to fibrinolysis were observed in many of these patients. Such changes are shown in Figure 1. Ten patients with erysipelas were tested whose plasma clot repeatedly remained resistant to fibrinolysis after twenty-four hours. In nine patients the resistance was observed to increase during the early period of the illness, later to remain undissolved after twenty-four hours. Three additional patients with erysipelas showed moderate or slight decrease in the resist- 
ance of their plasma clot to dissolution. Similar observations were noted in patients with other hemolytic streptococcal infections. Figure 2 shows the results of repeated studies of the resistance of the plasma of a patient with an acute tonsillitis. It is of interest to note that a few patients with undoubted infection with the hemolytic streptococcus failed to develop an enhanced resistance to fibrinolysis during or subsequent to their illness.

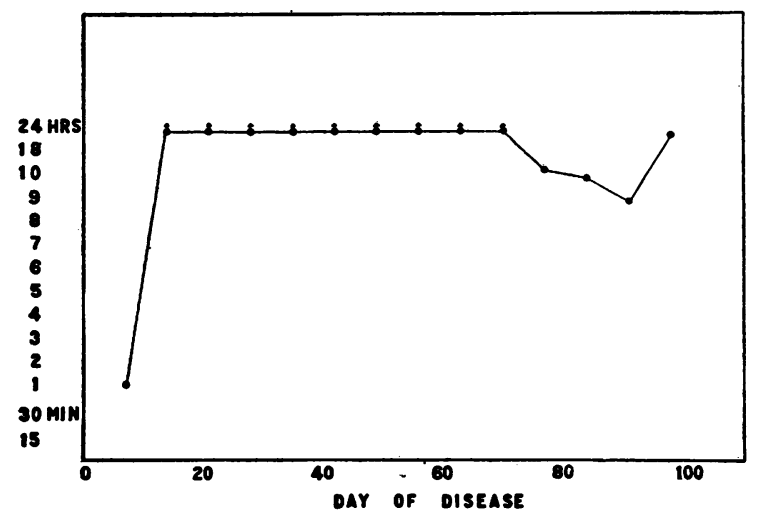

Fig. 2. The Changes in the Dissolution Time of the Plasma Clot of a Patient with Acute TonsILIITIS.

Tests were done on four patients with acute glomerular nephritis associated with infection with hemolytic streptococci. The plasma of none of these patients had "maximum resistance" to fibrinolysis.

Of the three patients with subacute bacterial endocarditis the plasma of two, from whose blood a streptococcus viridans was cultured, showed "maximum resistance" to fibrinolysis. From the third patient an "indifferent" streptococcus was obtained; the plasma of this patient was repeatedly highly susceptible to fibrinolysis.

One hundred and ninety-five samples of plasma from thirty-four patients with undoubted active rheumatic fever were tested. The results of these tests are summarized in Table I. Seventeen of the thirty-four patients gave adequate evidence for considering their illnesses as exacerbations of the rheumatic process. Twenty-eight of the thirty-four patients were studied at weekly intervals for four weeks or longer.

In twenty-nine of these patients, or 85.3 per cent, " maximum resistance", was encountered.
In Figure 3 are illustrated the results obtained in three illustrative patients. Of these twentynine patients, twenty were studied for periods varying between five and fifteen weeks. The samples of plasma from thirteen of the twenty patients were resistant to fibrinolysis for twentyfour hours persistently; Curve Number 1 in Figure 3 is an example of this group. Five additional patients showed variation in the resistance of their plasma as is illustrated by Curve No. 2 in Figure 3. In each of these the plasma resisted fibrinolysis for twenty-four hours when the patient was first seen and later dissolution was observed within the twenty-four hour period. In but one patient was the earlier sample of plasma dissolved after two and one-half hours; at all subsequent tests it remained unchanged after twentyfour hours, as had been observed in the acute hemolytic streptococcal infections.

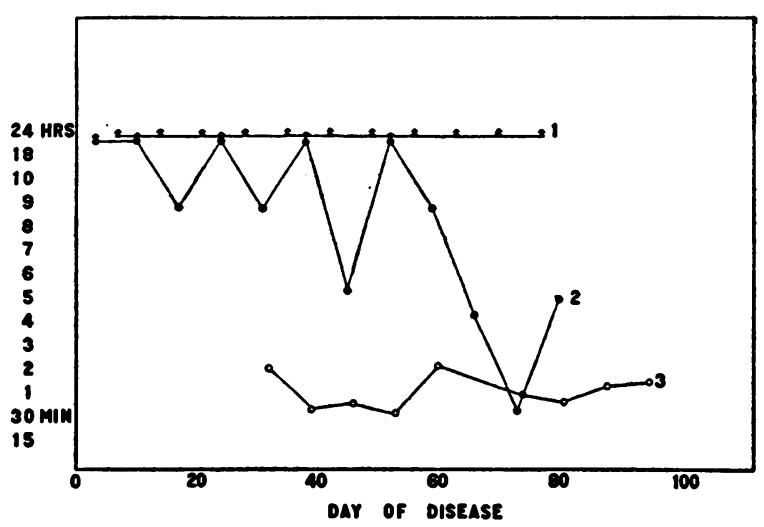

Fig. 3. The Changes in the Dissolution Time of the Plasma Clot of Three Patients with RheuMatic Fever.

As in patients with hemolytic streptococcal infections a few patients with rheumatic fever failed to show an enhanced resistance to fibrinolysis, even when followed for several weeks. The results of the tests of the plasma of one such patient are given in Figure 3.

Evidence was obtained in twenty-nine of the thirty-four patients of infection with the hemolytic streptococcus, either from a history of such an infection from one to eight weeks previous to, or as a result of, bacteriological studies at the time of the first anti-fibrinolytic test. In two patients whose plasma showed " maximum resistance" and in one each of those who are classified as 
showing "marked resistance," "definite resistance" and " high susceptibility," respectively, evidence of infection by the hemolytic streptococcus was lacking; there was no history or signs of such an infection and throat cultures did not reveal hemolytic streptococci.

In the eleven patients with active joint disease due to rheumatoid arthritis, forty-three samples of plasma were tested. The results are summarized in Table I. Of the two patients, samples of whose plasma were resistant to fibrinolysis for twenty-four hours, one was followed during an attack of acute sinusitis. Before the onset of the sinus infection the fibrin-clot was dissolved in two hours; the fibrin-clot became and remained resistant for twenty-four hours during the six weeks of observation. The observation of the antifibrinolytic properties of the plasma of these eleven patients was similar to the results obtained in the groups of individuals without infection with the hemolytic streptococcus.

\section{COMMENTS}

From the data presented, certain deductions are justifiable. It is evident that the blood plasma of normal individuals and patients without evidence of hemolytic streptococcal infection may contain antifibrinolysin in varying amounts. In a few individuals with infections due to microorganisms other than the hemolytic streptococcus, the resistance of the blood plasma to fibrinolysis may be high. In view of the observations that enhanced resistance to fibrinolysis may persist for an indeterminate time following a hemolytic streptococcal infection, it is possible that the patients with a high resistance may have had a previous hemolytic streptococcal infection some weeks before examination, in spite of the negative history. In the main, these results are in agreement with the observations of Tillett, Edwards and Garner (2) and Hadfield, Magee and Perry (3).

The blood plasma from patients with recent hemolytic streptococcal infections is generally much more resistant to fibrinolysis than that from control groups of patients. The variations observed are probably due to inherent differences in the reaction of the individual or to the variation in the ability of the infecting organism to call forth antifibrinolysins in man. That there are differences in the various strains of hemolytic streptococcus, depending upon their source and cultural characteristics, has been emphasized by Tillett (7), De Venter and Reich (8) and Hadfield, Magee and Perry (3). Inasmuch as different strains of hemolytic streptococci vary in their capacity to produce substances such as hemolysin and toxin, it is not surprising that they show differences in their fibrinolysin production.

We believe that the antifibrinolysis is an immune response to infection by hemolytic streptococci which are capable of producing fibrinolysin. The ability of these organisms to produce fibrinolysis may explain in part why the hemolytic streptococcus is so invasive in character, and it most certainly explains the character of the exudate that is seen early in the course of hemolytic streptococcal infection.

In view of the frequency with which rheumatic fever was preceded by infection with the hemolytic streptococcus in these cases, it was not surprising that the plasma from patients with rheumatic fever showed an enhanced resistance to fibrinolysis. The results in this group of patients with rheumatic fever here studied are in close agreement with those observed in patients with acute hemolytic streptococcal infection.

The plasma of patients with active rheumatoid arthritis gave results in all respects similar to that observed in patients without hemolytic streptococcal infection. In but one patient was there evidence of active infection by the hemolytic streptococcus. It would seem that the disease process in the majority of these patients was not associated or preceded by an active hemolytic streptococcal infection.

\section{SUMMARY AND CONCLUSIONS}

1. The plasma of patients with proven hemolytic streptococcal infection is more highly resistant to fibrinolysis than plasma from normal individuals and patients with infection caused by other microorganisms.

2. The resistance to fibrinolysis of the plasma of patients with rheumatic fever is comparable to that observed in patients with erysipelas and other acute hemolytic streptococcal infections.

3. Rheumatoid (atrophic) arthritis is not ac- 
companied by an increase in the antifibrinolytic property of the plasma.

We acknowledge our thanks to Miss Marjorie Jewell and Miss Eleanor Fleming for technical assistance.

\section{BIBLIOGRAPHY}

1. Tillett, W. S., and Garner, R. L., The fibrinolytic activity of hemolytic streptococci. J. Exper. Med., 1933, 58, 485.

2. Tillett, W. S., Edwards, L. B., and Garner, R. L., Fibrinolytic activity of hemolytic streptococci. The development of resistance to fibrinolysis following acute hemolytic streptococcus infections. J. Clin. Invest., 1934, 13, 47.

3. Hadfield, G., Magee, V., and Perry C. B., The lysis of fibrin by streptococci: Its application to the problems of rheumatic infection in children. Lancet, 1934, 1, 834.

4. Keefer, C. S., Myers, W. K., and Oppel, T. W., Streptococcal agglutinins in patients with rheumatoid (atrophic) arthritis and rheumatic fever. J. Clin. Invest., 1933, 12, 267.

5. Myers, W. K., Keefer, C. S., and Oppel, T. W., Skin reactions to nucleoprotein of streptococcus scarlatinae in patients with rheumatoid arthritis and rheumatic fever. J. Clin. Invest., 1933, 12, 279.

6. Myers, W. K., and Keefer, C. S., Antistreptolysin content of the blood serum in rheumatic fever and rheumatoid arthritis. J. Clin. Invest., 1934, $13,155$.

7. Tillett, W. S., Personal communication.

8. van DeVenter, J. K., and Reich, T., Antihuman fibrinolytic streptococci. Proc. Soc. Exper. Biol. and Med., 1934, 31, 821. 